\title{
Characterization of NDM-1-producing carbapenemase in Acinetobacter spp. and $E$. coli isolates from diseased pigs
}

\author{
Rongmin ZHANG, Yang WANG, Zhihai LIU, Jiyun LI, Wenjuan YIN, Lei LEI, Congming WU, \\ Jianzhong SHEN (ه)
}

Beijing Key Laboratory of Detection Technology for Animal-Derived Food Safety, College of Veterinary Medicine, China Agricultural University, Beijing 100193, China

\begin{abstract}
In recent years, the mobile metallo- $\beta$-lactamase (MBL) genes have been found to correspond to one of the most important resistance characters identified in Gramnegative bacteria, severely affecting clinical chemotherapy and threatening public health. The prevalence of mobile MBL genes and their flanking regions in Gram-negative bacteria from diseased pigs in China was investigated. A total of 334 lung samples from diseased pigs were screened for Gram-negative bacteria classified as non-susceptible to meropenem ( $\mathrm{MIC} \geqslant 4 \mathrm{mg} \cdot \mathrm{L}^{-1}$ ). Six isolates, including three Escherichia coli, two Acinetobacter baumanii and one A. calcoaeticus, exhibited MBL production and carried the $b l a_{\mathrm{NDM}-1}$ gene. S1-PFGE and Southern blot analysis showed that the $b l a_{\mathrm{NDM}-1}$ gene was located on the chromosome of one A. baumanii isolate and on plasmids of various sizes in the other five isolates. MIC testing using broth microdilution revealed that all $b l a_{\mathrm{NDM}-1}$-carrying isolates and some of their transconjugants exhibited resistance to almost all $\beta$-lactams tested. Whole genome sequencing revealed that the flanking region of the $b l a_{\mathrm{NDM}-1}$ gene from all porcine isolates had high levels of similarity with the corresponding regions in human isolates. One porcine $E$. coli isolate carrying $b l a_{\mathrm{NDM}-1}$ was typed as ST48, a common sequence type in human $E$. coli isolates. These results suggest the possibility of human-tofood animal transfer of $b l a_{\mathrm{NDM}-1}$-producing $E$. coli, highlighting the need for surveillance of carbapenemase producers among bacteria from food animals. In addition, the prudent use of antimicrobial agents to decrease the opportunities for co-selection of carbapenemase genes in food animals is also urgently needed.
\end{abstract}

Keywords carbapenemase, NDM-1, ISAba125, Enterobacteriaceae, food safety

Received May 22, 2015; accepted July 19, 2015

Correspondence: sjz@cau.edu.cn

\section{Introduction}

The emergence of metallo- $\beta$-lactamases (MBL; also known as class B carbapenemases), which hydrolyze all classes of $\beta$-lactams except monobactams, in Gramnegative human pathogens represents a major threat for clinical chemotherapy and public health ${ }^{[1]}$. The NDM-type carbapenemases, first identified in a strain of Klebsiella pneumoniae isolated in 2008 in New Delhi, India ${ }^{[2]}$, are one of the most important MBL types because of their clinical relevance and international dissemination across all continents except Antarctica ${ }^{[3]}$. To date, 16 variants of NDM (NDM-1 to NDM-16; no information on NDM-12, NDM-15 and NDM-16 is available in the GenBank database), which differ by one, two, or five amino acid substitutions at 14 positions (www.lahey.org/studies), have been identified mainly from Enterobacteriaceae isolated from nosocomial infections. In these NDM variants, the $b l a_{\mathrm{NDM}-1}$ gene is mostly located on conjugative plasmids, which facilitates its rapid dissemination ${ }^{[4]}$. Detailed analysis of the genetic environment of the $b l a_{\mathrm{NDM}-1}$ gene in the chromosome or on plasmids of various Gramnegative bacterial species revealed the presence of a conserved structure comprising the complete or truncated insertion sequence element ISAba125 and the bleomycin $\left(b l e_{\mathrm{MBL}}\right)$ resistance gene in the up- and down-stream regions of the $b l a_{\mathrm{NDM}-1}$ genes, respectively ${ }^{[4]}$.

Compared with the high prevalence of NDM in Gramnegative bacteria of human origin, there are only limited and sporadic reports of this type of carbapenemase in bacteria from non-human origins. In the environment, the $b l a_{\mathrm{NDM}-1}$ gene was first identified in multiple genera in 2011, including the opportunistic Enterobacteriaceae and non-fermentative Gram-negative bacteria, in the samples of both tap water and seepage water in New Deli, India ${ }^{[5]}$. The $b l a_{\mathrm{NDM}-1}$ gene was then found in $K$. pneumoniae isolated from a river in Vietnam ${ }^{[6]}$, as well as in 
Acinetobacter baumannii isolated from sewage of hospitals in Beijing, China ${ }^{[7]}$. Recently, bla $a_{\mathrm{NDM}-1^{-}}$-arrying $A$. calcoaceticus and $A$. junii were identified in environmental samples from livestock farms in China ${ }^{[8]}$. In animals, a single isolate of NDM-1-producing $A$. lwoffii and $A$. baumannii has been reported from a chicken and a pig, respectively ${ }^{[9,10]}$, and the $b l a_{\mathrm{NDM}-1}$ gene was also observed in clinical Escherichia coli isolates recovered from cats and dogs in the USA ${ }^{[11]}$.

As there have only been a few reports on the carbapenemases in bacteria from food animals, we screened the clinical samples of diseased pigs from an animal diagnostic laboratory in China to investigate the presence of the carbapenem resistance gene in Gramnegative bacteria, and further characterized the genetic environment of the carbapenem resistance gene.

\section{Materials and methods}

2.1 Bacterial isolation and identification, and phenotypic and molecular detection of MBL in Gram-negative bacteria

A total of 334 lung samples were collected from the animal diagnostic laboratory of Foshan University in Foshan City, Guangdong Province, China, between July and August 2013. Each sample was isolated from an individual animal and spread on a brain heart infusion (BHI) agar plate containing $2 \mathrm{mg} \cdot \mathrm{L}^{-1}$ meropenem (Ouhe Technology Company, Beijing, China) and $30 \mathrm{mg} \cdot \mathrm{L}^{-1}$ vancomycin, then incubated for $18 \mathrm{~h}$ at $37^{\circ} \mathrm{C}$. All colonies were selected and identified using Gram staining and sequence analysis of the 16S rDNA gene, using previously described primers $^{[12]}$. Species identification of Gram-negative bacteria was further conducted by MALDI-TOF MS (BrukerDaltonik GmbH, Bremen, Germany). The imipenemEDTA double-disc synergy test and E-test using MBL strips (bioMérieux, Craponne, France) were performed to screen for MBL production in these isolates. The MBLproducing isolates were screened for known mobile MBLencoding genes, NDM-, VIM-, SIM-, GIM-, AIM- and DIM-type $\beta$-lactamases were investigated using previously described PCR assays ${ }^{[10]}$.

\subsection{Conjugation assay}

Filter mating was performed with each of the original isolates using E. coli strain EC600 (a rifampicin-resistant strain) as the recipient. The original isolates and the recipient strains of $E$. coli EC600, both incubated overnight were adjusted to $0.5 \mathrm{McF}$ arland standard in BHI broth, and $5 \mu \mathrm{L}$ of parental and recipient strains were added to $1 \mathrm{~mL}$ of BHI broth and incubated for an additional $4 \mathrm{~h}$ at $37^{\circ} \mathrm{C}$, respectively. Then, $20 \mu \mathrm{L}$ of the parental strain and $60 \mu \mathrm{L}$ of the recipient strains were incubated together on a microporous membrane (Millipore, Bedford, MA,
USA) overnight for conjugation. The concentrations of antibiotics in the BHI agar plates used for the selection of transconjugants were $500 \mathrm{mg} \cdot \mathrm{L}^{-1}$ for rifampin and $1 \mathrm{mg} \cdot \mathrm{L}^{-1}$ for meropenem. Transconjugants were confirmed as MBL-producing isolates by PCR analysis, and pulsed-field gel electrophoresis (PFGE) was also performed to confirm that transconjugants were derivatives of the recipient strain EC600.

\subsection{Antimicrobial susceptibility testing}

The minimum inhibitory concentrations (MIC) of a range of antibiotics against the MBL-producing isolates, as well as their transconjugants, were determined using the broth microdilution method according to the Clinical and Laboratory Standards Institute document M100-S25 (2015) and the MIC were interpreted according to these standard $^{[13]}$. The E. coli isolate ATCC25922 was used for quality control.

\subsection{Molecular analysis of MBL-producing isolates}

The genetic relatedness of MBL-producing isolates was investigated by $X b a \mathrm{I}$-PFGE for E. coli and SmaI-PFGE for A. baumanii. The location of MBL genes in the original isolates was determined by S1-nuclease PFGE mapping and Southern blot analysis as described previously ${ }^{[14]}$. Multilocus sequence typing (MLST) of the MBL-producing isolates was performed according to published protocols (mlst.warwick.ac.uk/mlst/dbs/Ecoli).

2.5 Sequence, assembly, and annotation of the flanking region of the MBL gene

The total DNA of MBL-producing isolates was extracted using the Wizard $\AA$ Genomic DNA Purification kit (Promega, WI, USA), then subjected to whole genome sequencing. The library was constructed using the NEXT ${ }^{\circledR}$ Ultra $^{\mathrm{TM}}$ DNA Library Prep kit (New England Biolabs, Ipswich, UK), and paired-end sequencing was conducted on an Illumina Hiseq2500 (Berry Genomics Company, Beijing, China). A draft assembly of the sequences was generated using CLC Genomics Workbench 5 (CLC Bio, Aarhus, Denmark). All contigs were searched for $\beta$ lactamase genes using standalone Blast analysis ${ }^{[15]}$. Gap closure of the flanking regions of MBL genes was performed by PCR using a modified random primer walking strategy ${ }^{[16]}$. The putative coding sequences of the flanking region of MBL genes were obtained using ORF Finder programs (www.ncbi.nlm.nih.gov/gorf/orfig.cgi) and the Vector NTI program (Invitrogen, CA, USA).

\section{Results and discussion}

In this study, six isolates classified as non-susceptible to 
meropenem $\left(\mathrm{MIC} \geqslant 4 \mathrm{mg} \cdot \mathrm{L}^{-1}\right.$ ), obtained from six of the 334 lung samples from the diseased pigs, were confirmed as Gram-negative bacteria. These isolates were further identified by $16 \mathrm{~S}$ rRNA gene sequencing and the Bruker Daltonik MALDI Biotyper Classification system as E. coli $(n=3$, FSEC38, FSEC39, and FSEC69), A. baumanii $(n=$ 2, FSAB08 and FSAB62) and A. calcoaceticus $(n=1$, FSABC15). Imipenem-EDTA double-disc synergy tests and E-test MBL strip tests confirmed that the six isolates were MBL-positive, and PCR analysis using primers specific for mobile MBL genes revealed that all of the isolates were positive for $b l a_{\mathrm{NDM}}$. The $813 \mathrm{bp}$ nucleotide sequence of $b l a_{\mathrm{NDM}}$ in the six isolates showed $100 \%$ identity to that of the $b l a_{\mathrm{NDM}-1}$ gene on plasmid pKpANDM-1 of $K$. pneumoniae 05-506 (GenBank accession no. FN396876). The detection rate (1.8\%, $6 / 334)$ of the mobile MBL gene (bla $\left.a_{\mathrm{NDM}-1}\right)$ in the lung samples of diseased pigs in the current study was considerably higher than both that in the lung samples of pigs from commercial farms in Guangdong province during 2011-2012 (0.3\%, 1/313), and that in various samples (cloaca, rectum, nasal cavity, and lymph nodes) from commercial farms in Shandong province in 2012 $(0.3 \%, 1 / 396)^{[9,10]}$. To our knowledge, this is the first time that NDM-producing Enterobacteriaceae have been reported from food animals.

PFGE analysis of the three $b l a_{\mathrm{NDM}-1}$-carrying $E$. coli isolates and two bla $a_{\mathrm{NDM}-1}$-carrying $A$. baumanii isolates revealed distinct genomic heterogeneity in their $X b a \mathrm{I}$ and SmaI patterns, respectively. Comparison with the allelic profiles available in the aforementioned MSLT website identified E. coli isolate FSEC69 as sequence type (ST) 48. ST48 has been associated with hospital-acquired extendedspectrum $\beta$-lactamase (ESBL)-producing $E$. coli isolates from patients in Belgium ${ }^{[17]}$, and ESBL-producing E. coli isolates in fecal samples of healthy humans in Tunisia ${ }^{[18]}$. The sequence types of another two E. coli isolates FSEC38 and FSEC39 were novel and were designated as ST5084 and ST5069, respectively.

The six isolates exhibited resistance and high MIC to almost all $\beta$-lactam antibiotics tested, and only one E. coli isolate, FSEC39, showed borderline susceptibility to aztreonam $\left(4 \mathrm{mg} \cdot \mathrm{L}^{-1}\right)$. As the $b l a_{\mathrm{NDM}-1}$ gene cannot confer resistance to monobactams, a search for $\beta$-lactamase genes in the contigs of the two aztreonam-resistant $E$. coli isolates revealed that the AmpC $\beta$-lactamases genes $b a_{\mathrm{CMY}-2}+$ $b l a_{\text {DHA-1, }}$, and $b l a_{\text {ampC }}$ were observed in FSEC38 and FSEC69, respectively. Three genes had previously been identified as being responsible for plasmid-mediated aztreonam resistance in the Enterobacteriaceae ${ }^{[19]}$. It is noteworthy that no aztreonam-resistance gene was detected in the contigs of the three Acinetobacter isolates, suggesting that an unknown monobactam resistance gene may exist, a possibility which needs further investigation. Moreover, all isolates exhibited MICs of 2 to $256 \mathrm{mg} \cdot \mathrm{L}^{-1}$ to ciprofloxacin, which classified the isolates as resistant or intermediate. All isolates, except FSEC39, exhibited resistance to tetracycline, and had MIC for colistin of $\geqslant 4 \mathrm{mg} \cdot \mathrm{L}^{-1}$, while all isolates except FSAB62 exhibited resistance to gentamicin (Table 1).

S1-nuclease PFGE mapping and Southern blot analysis revealed that the gene $b l a_{\mathrm{NDM}-1}$ was located on varioussized plasmids, ranging from approximately $47-200 \mathrm{~kb}$, in five isolates and on the chromosomal DNA of $A$. baumanii FSAB08 (Table 1, Fig. 1). However, only the $b l a_{\mathrm{NDM}-1^{-}}$ carrying plasmids pEC39 and pEC69 were successfully transferred from FSEC39 and FSEC69, respectively, to $E$. coli EC600 using filter mating. Susceptibility tests revealed that both of the transconjugants presented resistance to all tested $\beta$-lactams except aztreonam and to gentamicin compared with recipient E. coli EC600, but remained susceptible to ciprofloxacin and colistin. In addition, one transconjugant EC600-69 also exhibited resistance to tetracycline and gentamicin and an elevated MIC for

Table 1 Antimicrobial susceptibility profiles of Acinetobacter. baumannii FSAB08 and FSAB62, A. calcoaceticus FSABC15, E. coli FSEC38, FSEC39, FSEC69, transconjugants EC600-39 and EC600-69, and recipient strains EC600

\begin{tabular}{|c|c|c|c|c|c|c|c|c|c|c|c|c|c|c|}
\hline \multirow{2}{*}{ Isolate } & \multirow{2}{*}{ pecies } & \multirow{2}{*}{$\begin{array}{l}\text { PFGE } \\
\text { type }\end{array}$} & \multirow{2}{*}{$\begin{array}{l}\text { MLST } \\
\text { type }\end{array}$} & \multicolumn{10}{|c|}{$\mathrm{MIC} /\left(\mathrm{mg} \cdot \mathrm{L}^{-1}\right)$} & \multirow{2}{*}{-Location of $b l a_{\mathrm{NDM}-1}$} \\
\hline & & & & IMP & MERO & $\mathrm{CTZ}$ & CAZ & $\mathrm{AZT}$ & CIP & GEN & $\mathrm{COL}$ & FFC & TET & \\
\hline$\overline{\text { FSAB08 }}$ & A. baumanii & 1 & - & 512 & 512 & $>1024$ & 256 & $>512$ & 2 & 64 & 128 & 8 & 32 & $\mathrm{C}$ \\
\hline FSAB62 & A. baumanii & 2 & - & 256 & 256 & 1024 & $>1024$ & 64 & 128 & 0.125 & 4 & 256 & 512 & $\mathrm{P} / \mathrm{pAB} 62(\sim 47 \mathrm{~kb})$ \\
\hline FSABC15 & A. calcoaceticus & - & - & 256 & 512 & 1024 & $>1024$ & 128 & 256 & 128 & 4 & 512 & $>512$ & $\mathrm{P} / \mathrm{pABC} 15(\sim 47 \mathrm{~kb})$ \\
\hline FSEC38 & E. coli & a & 5084 & 64 & 32 & 1024 & $>1024$ & 512 & 2 & 128 & 8 & 512 & 256 & $\mathrm{P} / \mathrm{pEC} 38(\sim 50 \mathrm{~kb})$ \\
\hline FSEC39 & E. coli & $\mathrm{b}$ & 5069 & 128 & 128 & 512 & $>1024$ & 4 & 8 & $>512$ & 2 & 32 & 4 & P/pEC39 ( 70 kb) \\
\hline EC600-39 & E. coli & - & - & 32 & 32 & 128 & 1024 & 0.25 & 0.125 & 512 & 0.5 & 4 & 4 & $\mathrm{P} / \mathrm{pEC} 39(\sim 70 \mathrm{~kb})$ \\
\hline FSEC69 & E. coli & $\mathrm{c}$ & 48 & 128 & 256 & $>1024$ & $>1024$ & 16 & 128 & 256 & 4 & 512 & 256 & P/pEC69 $(\sim 200 \mathrm{~kb})$ \\
\hline EC600-69 & E. coli & - & - & 64 & 64 & 256 & $>1024$ & 0.125 & 0.25 & 64 & 0.5 & 512 & 64 & P/pEC69 $(\sim 200 \mathrm{~kb})$ \\
\hline EC600 & E. coli & - & - & 0.25 & 0.03 & 2 & 0.5 & 0.125 & 0.015 & 1 & 0.25 & 4 & 2 & - \\
\hline
\end{tabular}

Note: IMP, imipenem; MERO, meropenem; CTZ, ceftizoxime; CAZ, ceftazidime; AZT, aztreonam; CIP, ciprofloxacin; GEN, gentamicin; COL, colistin; FFC, florfenicol; TET, tetracycline. 
florfenicol. These results indicate that the determinants conferring resistance to three antimicrobials other than $\beta$ lactams can be co-transferred with the $b l a_{\mathrm{NDM}-1}$ gene from the donor to the recipient E. coli EC600 (Table 1).

Analysis of the flanking regions of the $b l a_{\mathrm{NDM}-1}$ gene on two plasmids and in one chromosome of three Acinetobacter isolates revealed that the $b l a_{\mathrm{NDM}-1}$ gene was located in an identical $9923 \mathrm{bp}$ fragment, which exhibited 100\% nucleotide sequence identity to the corresponding region of plasmid pNDM-BJ01 (JQ001791) isolated from human clinical A. lwoffii strain WJ10621 from Beijing, China ${ }^{[20]}$. In addition, a 5029 bp region including the $b l a_{\mathrm{NDM}-1}$ gene, its upstream ISAba125 and aminoglycoside resistance gene aphA6, and its downstream gene ble $e_{\mathrm{MBL}}$ also had 99.9\% (5017/5029) nucleotide sequence identity to that in plasmid pAL-01 (JN616388) in chicken A. lwoffii strain SGC-HZ9 (Fig. 2) ${ }^{[10]}$. Further downstream of the ble $e_{\mathrm{MBL}}$ gene, a truncated putative phosphoribosylanthranilate isomerase gene $\operatorname{trpF}$ was identified, followed by $d s b C$, cutA1, groES and groEL, and insE, encoding the oxidoreductase protein, then tolerance protein, heat chaperonin protein and transposase, respectively.

In the $b l a_{\mathrm{NDM}-1}$-carrying regions in plasmids from porcine E. coli isolates, the nucleotide sequences of a $15914 \mathrm{bp}$ segment of pEC38 (GenBank accession no. KT164808), a 10384 bp fragment of pEC39 and a 6406 bp fragment of pEC69 (GenBank accession no. KT164809) were obtained by whole genome sequencing and primer walking (Fig. 2). This $15.9 \mathrm{~kb} b l a_{\mathrm{NDM}-1}$-harboring region in pEC38 had $100 \%$ nucleotide identity with the corresponding region of plasmid pKpn-SX04 (accession no. NG_041664) from $K$. pneumoniae derived from a patient from the program of Chinese Antimicrobial Resistance Surveillance of Nosocomial Infections in
$2012^{[21]}$. In both cases, a disrupted ISAba125 ( $\triangle$ IS Aba125) resulted from the insertion of another insertion sequence, the IS5 element. Small differences between these two plasmids existed: a truncation of the left direct target site (5'-CTAA-3') and the 234 bp ISAba125 sequence was observed in pEC38. However, it was reported that the $7830 \mathrm{bp}$ fragment, namely the $3^{\prime}$ region of the $\triangle \mathrm{ISAba125}$, bla $_{\mathrm{NDM}-1}$, ble $_{\mathrm{MBL}}, \operatorname{trpF}, d s b C$, cutA1, groES, groEL, and insE, facilitated the horizontal mobilization of the $b l a_{\mathrm{NDM}-1}$ gene among Enterobacteriaceae ${ }^{[21]}$.

Both the $9693 \mathrm{bp}$ and $5340 \mathrm{bp}$ bla $a_{\mathrm{NDM}-1}$-carrying fragments of pEC39 and pEC69 had $>99.9 \%$ nucleotide sequence identity with the corresponding region of plasmid pGUE-NDM (accession no. JQ364967) from E. coli isolate GUE, a community-acquired strain from India (Fig. 2) ${ }^{[22]}$. Downstream of the bla $a_{\mathrm{NDM}-1}$ gene, the ble $e_{\mathrm{MBL}}$, $\operatorname{trpF}$, and $d s b C$ genes were observed in $\mathrm{pEC} 39$, followed by the insertion sequence ISCRI, and by a class 1 integron structure carrying three gene cassettes, including streptomycin/spectinomycin resistance gene aadA2, the gene orf $F$ for a hypothetical protein, and the trimethoprim resistance gene $d f_{r} A 12$. The sequence further downstream of int 1 also had a high degree of nucleotide identity (99.9\%) to IS26 present in pGUE-NDM. Instead of the intact or truncated ISAba125 element observed immediately upstream of the $b l a_{\mathrm{NDM}-1}$ gene in other cases in this study, a rifampicin resistance gene aar-3 and chloramphenicol resistance gene catB3 were observed in pEC69 (Fig. 2).

\section{Conclusions}

The results of this study confirm that $b l a_{\mathrm{NDM}-1}$ is the only mobile MBL gene present in Acinetobacter spp. and E. coli
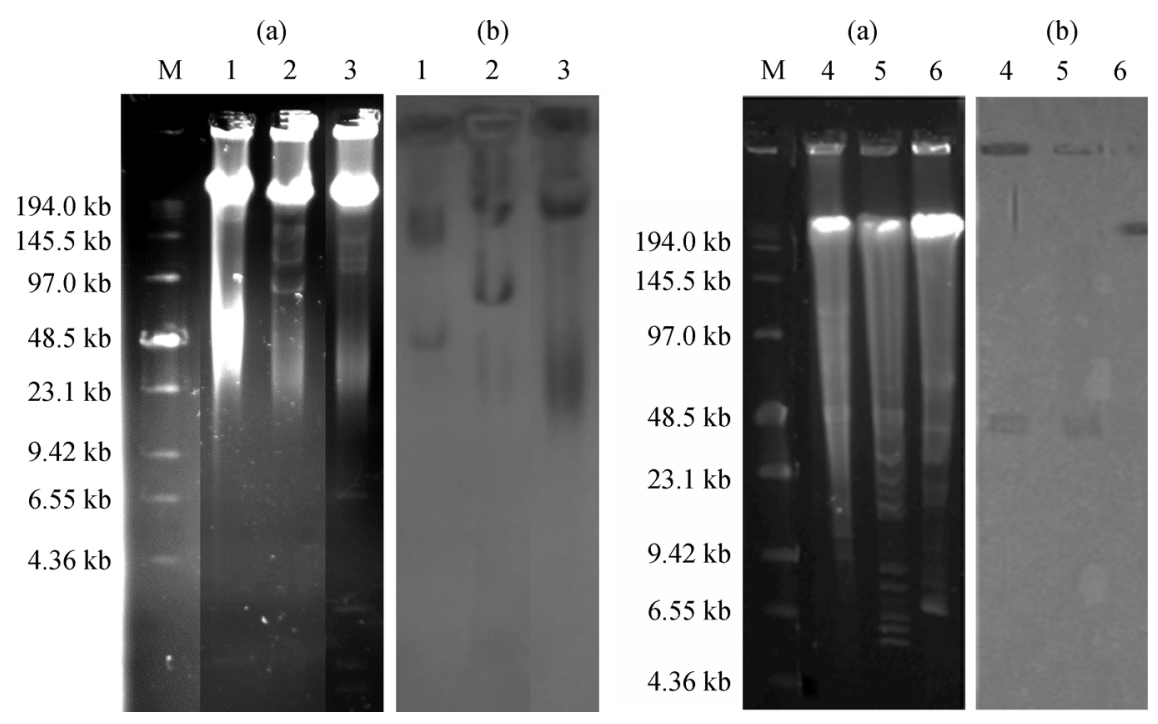

Fig. 1 Localization of $b l a_{\mathrm{NDM}-1}$ in NDM-1-producing isolates by S1-PFGE (a) and Southern blot hybridization with $b l a_{\mathrm{NDM}-1}$ probe (b). Lane M, low-range pulsed-field gel marker (New England BioLabs, Beverly, MA). Lane 1-3, E. coli isolates FSEC38, FSEC39 and FSEC69; lane 4, Acinetobacter. calcoaceticus isolate FSABC15; lane 5-6, A. baumanii isolates FSAB62 and FSAB08. 

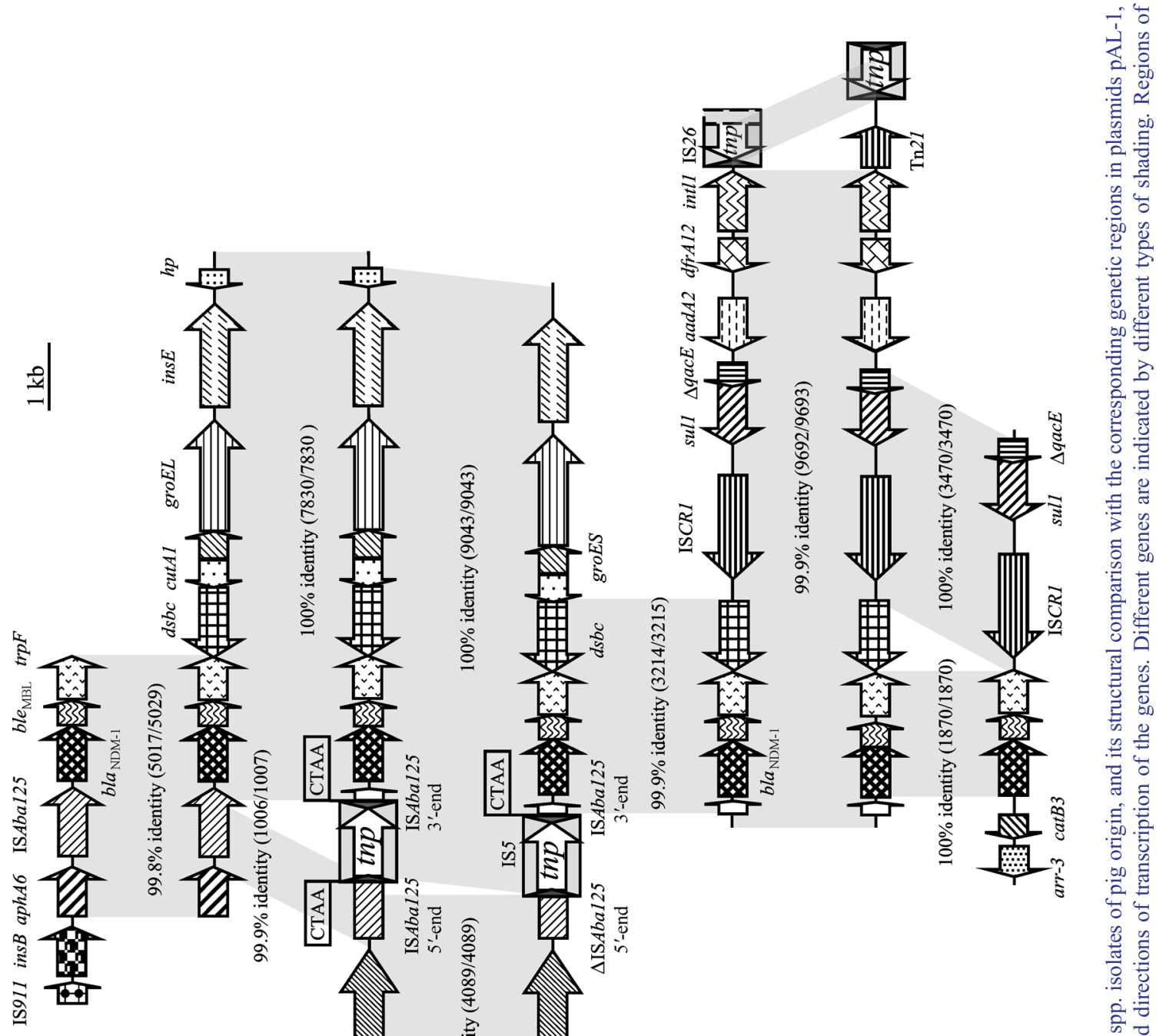

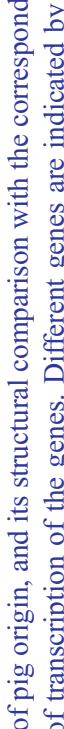




isolates from food animals. Similar to the spread of $b l a_{\mathrm{NDM}-1}$ in bacteria of clinical origin, both the plasmid and insertion sequence elements, such as ISAba125, IS5 and IS26, may be important in the dissemination of the $b l a_{\mathrm{NDM}-1}$ gene and its conserved flanking regions in bacteria from food animals. Moreover, the frequency of identification of ESBL-carrying E. coli ST48 from both human fecal and clinical samples, as well as the high level of similarity of the genetic environment of the $b l a_{\mathrm{NDM}-1}$ gene from the porcine $E$. coli isolate found in human Enterobacteriaceae, suggests the possibility of human-tofood animal transfer of this $b l a_{\mathrm{NDM}-1}$-producing isolate. Thus, enhanced and continued efforts are needed to monitor carbapenemase producers in bacteria from food animals. It should be noted that carbapenems are not approved for use in food animals, and the $b l a_{\mathrm{NDM}-1}$ gene was found to coexist with other genes conferring resistance to aminoglycosides, phenicols, and tetracyclines in isolates in this study, the co-selection and co-transfer of carbapenemase genes under the selective pressure imposed by these genes seems be key to a major role in their dissemination. Therefore, the prudent use of antimicrobial agents to decrease the opportunities for co-selection of carbapenemase genes in food animals is urgently needed.

Acknowledgements This study was supported by grants from the National Basic Research Program of China (2013CB127200), and the National Natural Science Foundation of China (31422055).

Compliance with ethics guidelines Rongmin Zhang, Yang Wang, Zhihai Liu, Jiyun Li, Wenjuan Yin, Lei Lei, Congming Wu, and Jianzhong Shen declare that they have no conflict of interest or financial conflicts to disclose.

All applicable institutional and national guidelines for the care and use of animals were followed.

\section{References}

1. Cornaglia G, Giamarellou H, Rossolini G M. Metallo- $\beta$-lactamases: a last frontier for $\beta$-lactams? The Lancet Infectious Diseases, 2011, 11(5): 381-393

2. Yong D, Toleman M A, Giske C G, Cho H S, Sundman K, Lee K, Walsh T R. Characterization of a new metallo- $\beta$-lactamase gene, $b l a_{\mathrm{NDM}-1}$, and a novel erythromycin esterase gene carried on a unique genetic structure in Klebsiella pneumoniae sequence type 14 from India. Antimicrobial Agents and Chemotherapy, 2009, 53(12): 5046-5054

3. Satlin M J, Calfee D P, Chen L, Fauntleroy K A, Wilson S J, Jenkins S G, Feldman E J, Roboz G J, Shore T B, Helfgott D C, Soave R, Kreiswirth B N, Walsh T J. Emergence of carbapenem-resistant Enterobacteriaceae as causes of bloodstream infections in patients with hematologic malignancies. Leukemia \& Lymphoma, 2013, 54 (4): 799-806

4. Dortet L, Poirel L, Nordmann P. Worldwide dissemination of the NDM-type carbapenemases in Gram-negative bacteria. BioMed Research International, 2014: 249856

5. Walsh T R, Weeks J, Livermore D M, Toleman M A. Dissemination of NDM-1 positive bacteria in the New Delhi environment and its implications for human health: an environmental point prevalence study. The Lancet Infectious Diseases, 2011, 11(5): 355-362

6. Isozumi R, Yoshimatsu K, Yamashiro T, Hasebe F, Nguyen B M, Ngo T C, Yasuda S P, Koma T, Shimizu K, Arikawa J. bla ${\mathrm{NDM}-1^{-}}^{-}$ positive Klebsiella pneumoniae from environment, Vietnam. Emerging Infectious Diseases, 2012, 18(8): 1383-1385

7. Zhang C, Qiu S, Wang Y, Qi L, Hao R, Liu X, Shi Y, Hu X, An D, Li Z, Li P, Wang L, Cui J, Wang P, Huang L, Klena J D, Song H. Higher isolation of NDM-1 producing Acinetobacter baumannii from the sewage of the hospitals in Beijing. PLoS ONE, 2014, 8(6): e64857

8. Wang B, Sun D. Detection of NDM-1 carbapenemase-producing Acinetobacter calcoaceticus and Acinetobacter junii in environmental samples from livestock farms. Journal of Antimicrobial Chemotherapy, 2015, 70(2): 611-613

9. Zhang W J, Lu Z, Schwarz S, Zhang R M, Wang X M, Si W, $\mathrm{Yu}$ S, Chen L, Liu S. Complete sequence of the bla $a_{\mathrm{NDM}-1}$-carrying plasmid pNDM-AB from Acinetobacter baumannii of food animal origin. Journal of Antimicrobial Chemotherapy, 2013, 68(7): 16811682

10. Wang Y, Wu C, Zhang Q, Qi J, Liu H, Wang Y, He T, Ma L, Lai J, Shen Z, Liu Y, Shen J. Identification of New Delhi metallo- $\beta$ lactamase 1 in Acinetobacter lwoffii of food animal origin. PLoS ONE, 2012, 7(5): e37152

11. Shaheen B W, Nayak R, Boothe D M. Emergence of a New Delhi metallo- $\beta$-lactamase (NDM-1)-encoding gene in clinical Escherichia coli isolates recovered from companion animals in the United States. Antimicrobial Agents and Chemotherapy, 2013, 57(6): 2902-2903

12. Wang Y, He T, Schwarz S, Zhao Q, Shen Z, Wu C, Shen J. Multidrug resistance gene $c f r$ in methicillin-resistant coagulasenegative staphylococci from chickens, ducks, and pigs in China. International Journal of Medical Microbiology, 2013, 303(2): 8487

13. CLSI document M100-S25. Performance standards for antimicrobial susceptibility testing; twenty-fifth informational supplement. Panama: Clinical and Laboratory Standards Institute, 2015

14. Wang Y, Wang X, Schwarz S, Zhang R, Lei L, Liu X, Lin D, Shen J. IMP-45-producing multidrug-resistant Pseudomonas aeruginosa of canine origin. Journal of Antimicrobial Chemotherapy, 2014, 69(9): 2579-2581

15. Benson D A, Cavanaugh M, Clark K, Karsch-Mizrachi I, Lipman D J, Ostell J, Sayers E W. GenBank. Nucleic Acids Research, 2013, 41 (D1): D36-D42

16. Liu Y, Wang Y, Schwarz S, Li Y, Shen Z, Zhang Q, Wu C, Shen J. Transferable multiresistance plasmids carrying cfr in Enterococcus spp. from swine and farm environment. Antimicrobial Agents and Chemotherapy, 2013, 57(1): 42-48

17. Smet A, Martel A, Persoons D, Dewulf J, Heyndrickx M, Claeys G, Lontie M, Van Meensel B, Herman L, Haesebrouck F, Butaye P. Characterization of extended-spectrum $\beta$-lactamases produced by Escherichia coli isolated from hospitalized and nonhospitalized patients: emergence of CTX-M-15-producing strains causing urinary tract infections. Microbial Drug Resistance, 2010, 16(2): 129-134 
18. Ben Sallem R, Ben Slama K, Estepa V, Jouini A, Gharsa H, Klibi N, Sáenz Y, Ruiz-Larrea F, Boudabous A, Torres C. Prevalence and characterisation of extended-spectrum beta-lactamase (ESBL)producing Escherichia coli isolates in healthy volunteers in Tunisia. European Journal of Clinical Microbiology \& Infectious Diseases, 2012, 31(7): 1511-1516

19. Philippon A, Arlet G, Jacoby G A. Plasmid-determined AmpC-type $\beta$-lactamases. Antimicrobial Agents and Chemotherapy, 2002, 46 (1): $1-11$

20. Hu H, Hu Y, Pan Y, Liang H, Wang H, Wang X, Hao Q, Yang X, Yang X, Xiao X, Luan C, Yang Y, Cui Y, Yang R, Gao G F, Song Y,
Zhu B. Novel plasmid and its variant harboring both a $b l a_{\mathrm{NDM}-1}$ gene and type IV secretion system in clinical isolates of Acinetobacter lwoffii. Antimicrobial Agents and Chemotherapy, 2012, 56(4): 1698-1702

21. Wang X, Xu X, Li Z, Chen H, Wang Q, Yang P, Zhao C, Ni M, Wang H. An outbreak of a nosocomial NDM-1-producing Klebsiella pneumoniae ST147 at a teaching hospital in mainland China. Microbial Drug Resistance, 2014, 20(2): 144-149

22. Bonnin R A, Poirel L, Carattoli A, Nordmann P. Characterization of an IncFII plasmid encoding NDM-1 from Escherichia coli ST131. PLoS ONE, 2012, 7(4): e34752 